\title{
Preparing for infectious disease threats at mass gatherings: the case of the Vancouver 2010 Olympic Winter Games
}

\author{
Kamran Khan MD MPH, Clark C. Freifeld BS, Jun Wang MSc, Sumiko R. Mekaru DVM MPVM, \\ David Kossowsky BA, Amy L. Sonricker MPH, Wei Hu BSC, Jennifer Sears BSc, Angie Chan MPA, \\ John S. Brownstein PhD
}

Previously published at www.cmaj.ca

$\mathrm{W}$ ith the global population approaching seven billion and international access to commercial air travel expanding, the number, frequency and scale of human congregations has increased dramatically during the past half century. Today, mass gatherings of hundreds of thousands to millions of people from all corners of the globe have become common. Such gatherings are held for a multitude of reasons: religious (e.g., the Hajj), political (e.g., Group of 20 [G-20] summits), socio-cultural (e.g., World Pride) and sports-related (e.g., Olympic Games), to name a few. ${ }^{1}$ Despite their importance, mass gatherings carry the risk of locally amplifying and subsequently disseminating infectious disease threats around the world. ${ }^{2}$ When travellers attend a mass gathering, they may unknowingly introduce infectious agents acquired in their home environments. In settings conducive to the spread of infection, epidemics among attendees and their contacts may ensue. ${ }^{3,4}$ Those who are exposed may subsequently transport the infectious agents internationally, spawning new epidemics in other parts of the world.

Current efforts to prepare for infectious disease threats at mass gatherings are generally led by the host country, often in collaboration with international public health agencies. ${ }^{2}$ Such efforts employ strategies that are directed, for the most part, at the site of the gathering. These may include enhanced surveillance, infection control measures to minimize the transmission of disease and ensuring the availability of resources to enable rapid response to an epidemic, should one arise. However, these efforts do not typically take into account a broader understanding of the populations attending the mass gathering and the risks associated with infectious disease threats at their points of departure. We propose a conceptual model that could complement existing preparedness efforts by expanding the geographic perspective of public health surveillance worldwide at a time when large numbers of people from around the globe are travelling to attend a mass gathering. Using this model would provide public health experts with the opportunity to identify and deal with an infectious disease threat at its source and, failing that, at the site of the mass gathering. This approach could potentially prevent importation of infection by persons travelling to the site of the mass gathering and/or exportation of infection as attendees at the gathering return home.

\section{Key points}

- Mass gatherings are common events with the potential to locally amplify and subsequently disseminate infectious disease threats around the world.

- Current efforts to prepare for infectious disease threats at mass gatherings focus primarily on interventions at the sites of such gatherings.

- Real-time integration of Web-based infectious disease surveillance with knowledge of worldwide patterns of commercial air traffic could significantly enhance global situational awareness of infectious disease threats.

Recent innovations in public health surveillance, such as the Canadian-based Global Public Health Intelligence Network, permit real-time global situational awareness (by leveraging media reports on the Internet) that typically outperforms traditional surveillance efforts and that exists outside politically influenced communication channels. By integrating knowledge of worldwide patterns of commercial air traffic ${ }^{6-8}$ with global surveillance of infectious diseases via Web-based intelligence-gathering tools, ${ }^{9,10}$ the conceptual model presented here seeks to identify locations in the world from where large numbers of people are expected to travel to attend a mass gathering and where infectious disease threats of public health significance are reported. This integrated knowledge could be used to notify officials at the site of the gathering of an emerging international threat and could foster a culture of greater international cooperation by motivating cities and countries that share common risks of infectious disease threats to work collaboratively. Herein, we apply our conceptual model to the Vancouver 2010 Olympic and Paralympic Winter Games.

From the Centre for Research on Inner City Health, Keenan Research Centre, Li Ka Shing Knowledge Institute (Khan, Wang, Kossowsky, Hu, Sears, Chan), and the Department of Medicine, Division of Infectious Diseases (Khan) St. Michael's Hospital, Toronto, Ont.; the Department of Health Policy, Management and Evaluation (Khan), University of Toronto, Toronto, Ont.; the Computational Epidemiology Group, Children's Hospital Informatics Program (Freifeld, Mekaru, Sonricker, Brownstein), and the Department of Pediatrics, Harvard Medical School (Brownstein), Children's Hospital Boston, Boston, USA; Media Laboratory (Freifeld), Massachusetts Institute of Technology, Cambridge, USA; and School of Public Health (Mekaru), Boston University, Boston, USA

CMAJ 2010. DOI:10.1503/cmaj.100093 


\section{Developing the model}

\section{Global air travel}

The BIO.DIASPORA Project is a multidisciplinary collaboration that is dedicated to studying the mobility of the world's population and its implications for global health, security and prosperity (www.biodiaspora.com). A core objective of this project is to understand the global interconnectedness and interdependence that are facilitated by the worldwide airline transportation network. ${ }^{6}$ To accomplish this, city-level analyses are performed using commercial air traffic data from more than 3500 airports, nearly 700 airlines and over two billion passenger trips every year. For passenger trips, information on the complete flight itineraries of travellers, including their connecting flights, is analyzed. However, these data do not include information about the characteristics of individual passengers or about their ground travel.

In the case of the 2010 Olympic Winter Games, we first analyzed worldwide air traffic patterns for February 2007 and 2008 to predict the leading origins of passengers who would be travelling to Vancouver in February 2010 (we did not have access to data for February 2009). We found a high degree of consistency between 2007 and 2008 in the global origins and volumes of passengers travelling into Vancouver. In February 2008, international passengers travelled to Vancouver from more than 800 cities worldwide; however, nearly two-thirds of them originated from just 25 cities. For the current analysis, we concentrated infectious disease surveillance in those 25 cities.
Next, we performed analyses to estimate the size of any potential surge in international air traffic that might coincide with the Olympic Winter Games in Vancouver. To accomplish this, we analyzed historical trends in international air traffic over the past decade, focusing on the Olympic Winter Games held in Torino, Italy, in February 2006. We did not use results from our analysis of the 2002 Olympic Winter Games in Salt Lake City, USA, as this event took place shortly after the Sept. 11, 2001, terrorist attacks in the United States and was thought to be poorly representative of the Olympic Games in Vancouver. To estimate the size of the surge, we performed time series analysis using an autoregressive integrated moving-average model. We estimated surges in international passenger traffic above expected seasonal levels for Torino and three of its neighbouring cities: $29.7 \%$ in Torino, 9.3\% in Genoa, 6.1\% in Bologna and 4.0\% in Milan. Together, these surges represented nearly 120000 passenger trips. If a surge of similar size were experienced in Vancouver, it would account for less than one-fifth (18\%) of the city's total volume of international passenger traffic in February 2010 (see Figure 1). We did not attempt to predict the geographic origins of these additional passengers, since the experience from Torino might not be generalizable to Vancouver in this regard.

Finally, we used data from flight schedules and network analysis to calculate the time required to travel from all commercial airports worldwide to Vancouver in February 2010, using the shortest path available. For pathogens with known

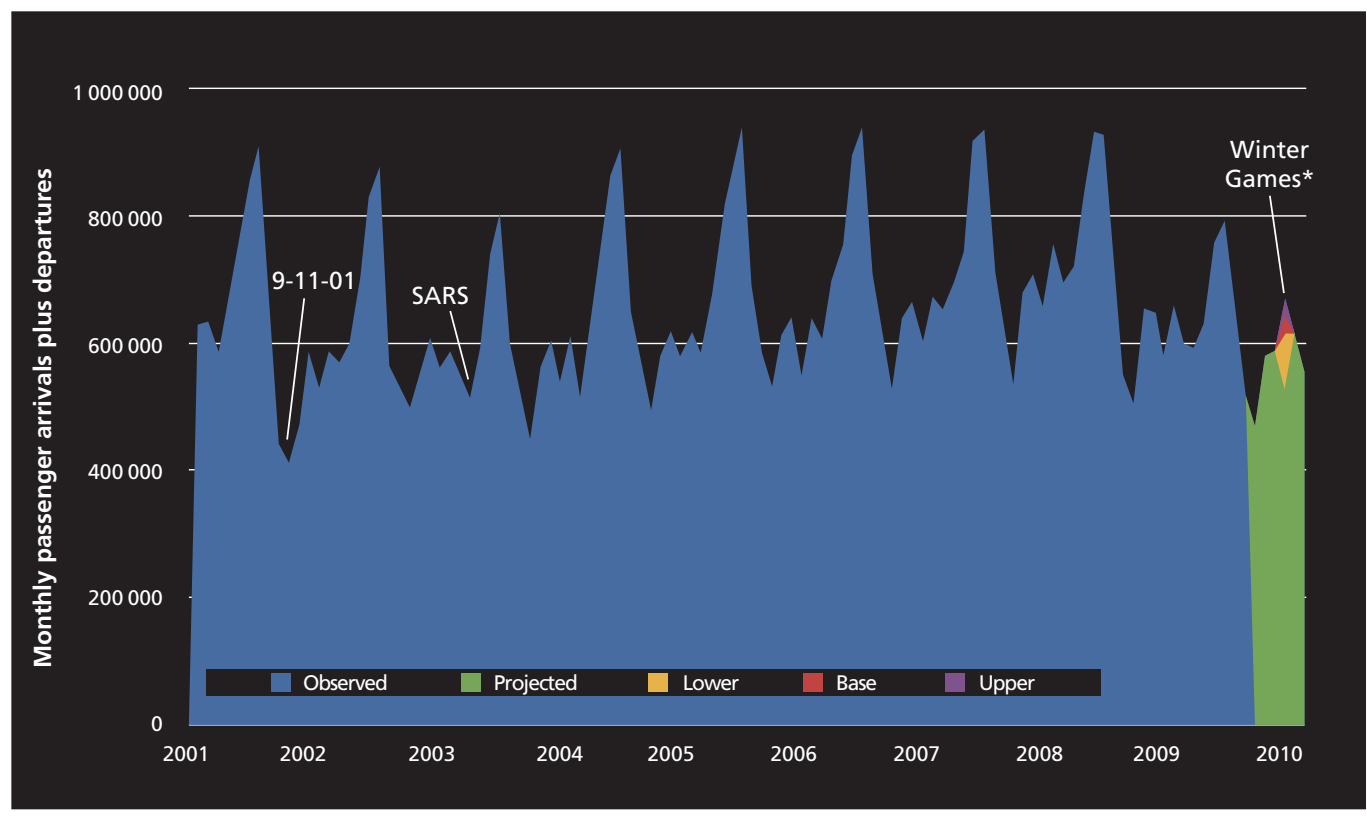

Figure 1: Predicted surge in international passenger traffic in Vancouver during the 2010 Olympic Winter Games. This figure depicts monthly flows of international passengers travelling through Vancouver International Airport during the past decade. Traffic flows through December 2009 reflect actual data, whereas flows during the first quarter of 2010 are predicted values based on time series analysis. The size of the predicted surge is proportional to the surge in international passenger traffic observed in Italy during the 2006 Olympic Winter Games in Torino (i.e., base estimate). Lower and upper estimates are also shown, representing 0.5 and 1.5 times the size of the surge observed during the 2006 Games in Torino. The significant reduction in baseline passenger flow observed in 2009 is consistent with that observed elsewhere in the world and is probably related to changes in the global economy. SARS = severe acute respiratory syndrome. 
incubation periods, information on travel times to Vancouver could be used to consider the role of screening travellers upon arrival at Vancouver International Airport. Although the effectiveness of infrared thermography or other screening modalities remains unproven, ${ }^{11}$ the value of screening on entry should theoretically be greatest for pathogens with short incubation periods that originate in cities requiring a long duration of travel to reach Vancouver. This is because longer travel durations offer greater opportunities for persons with latent infection to transition to symptomatic illness during the course of their trips.

\section{Global surveillance for infectious diseases}

HealthMap is an online resource that monitors a wide range of information sources for reports and warnings of outbreaks of infectious disease at the local or regional level. ${ }^{12}$ An automated text-processing system evaluates more than 30000 sources for disease outbreaks and their associated locations and maps the information to an interactive display (see www.healthmap.org). For the Vancouver Olympics, we sought to increase the sensitivity of the automated system for small outbreaks of disease in the 25 cities of interest mentioned above. We used a subsequent manual review to ensure specificity. To accomplish this dual objective, we took advantage of the widespread availability of RSS (really simple syndication) feeds to construct an automatically updating data set of news stories about infectious diseases. RSS feeds are Webbased feed formats used to notify subscribers of updates from frequently published Internet sources, such as news sites. We constructed a list of over 200 freely available RSS feeds associated with local television stations and newspapers, with particular attention to small neighbourhood media. We subjected all news stories to a multistep inclusion and exclusion process. Articles that contained prespecified disease words (e.g., "measles," "polio") were automatically included in the data set. We evaluated stories without those words for more

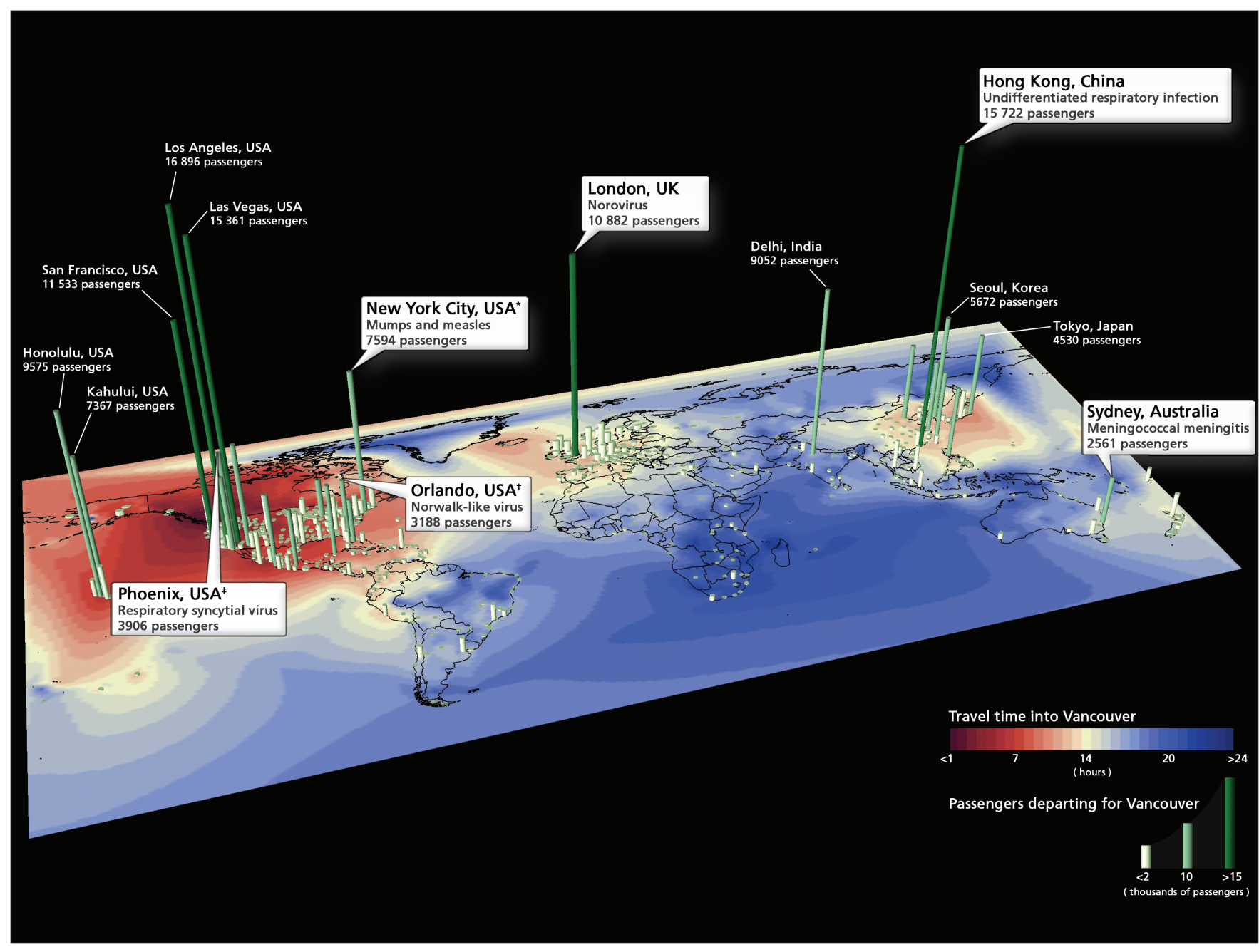

Figure 2: Potential infectious disease threats to Vancouver during the 2010 Olympic Winter Games. This figure depicts a selected number of infectious disease threats detected in February 2010 via the Internet, alongside cities with historically high volumes of passengers departing for Vancouver during the month of February. An up-to-date list of potential infectious disease threats can be viewed at www.healthmap.org/olympics. *Mumps and measles cases are reported for multiple locations across New Jersey and New York State, USA. †An outbreak of a Norwalk-like viral illness has been reported in Flagler County, Florida, USA. ¥An outbreak of respiratory syncytial virus has been reported in Mesa, Arizona, USA. 
general disease terms (e.g., "sick," "ill" or "death"). We then evaluated articles meeting the general-term inclusion criteria for exclusion terms (e.g., "crash," "shooting," "police") to decrease the proportion of news stories about events not related to disease. We deliberately kept the exclusion list short to reduce the risk of missing an important story. Frequent polling of large numbers of RSS feeds requires substantial computing time, primarily because of network latency. Furthermore, the dependence of this type of analysis on human review of stories in the data set would restrict the usefulness of this approach at a global scale. Nevertheless, for a limited number of locations and finite periods of elevated risk, this approach offers flexibility and timeliness.

\section{Integrated risk assessment}

We used the geographically directed surveillance described above to identify potential infectious disease threats at locations around the world that have a high influx of passengers into Vancouver. The analysis was performed on an hourly basis leading up to and during the course of the 2010 Olympic Winter Games (see Figure 2). In this analysis we reported threats (a) with high capability of spreading from person to person via respiratory transmission or direct contact, (b) that involved dangerous drug-resistant pathogens and (c) that involved pathogens that could be indicative of an act of bioterrorism. To illustrate this approach to risk assessment, we created a prototype analysis (www.healthmap.org /olympics) that generated real-time news of infectious disease threats in cities that historically have been strongly connected to Vancouver during the month of February.

\section{Policy considerations}

During the 1991 Special Olympics World Summer Games in the United States, an outbreak of measles was triggered by an infected athlete from Argentina, where a measles epidemic was under way. ${ }^{13}$ That incident supports the concept that dangerous infectious diseases can be imported to large sporting events and other mass gatherings, spawning local epidemics and leading to subsequent international dissemination. The conceptual model presented here could have utility in such settings, by facilitating the detection of disease threats at international locations with a high degree of connectivity to the city hosting the gathering, specifically when the gathering is set to occur. This information could offer the host city an opportunity to mobilize a response at the site of the mass gathering and/or to work collaboratively with other countries to confront threats upstream, at their international sources. However, decisions to act must take a variety of factors into consideration, such as the apparent seriousness of the threat, the ability of the threat to produce an epidemic that could be disseminated internationally by departing travellers, the probability that the threat could lead to an epidemic at the site of the mass gathering, the health and human resources needed to mobilize an effective response and the potential opportunity costs associated with the consumption of finite resources.

In our analysis, we identified no threats that were deemed to pose a serious risk to the 2010 Olympic Winter Games.
However, if a serious threat had been identified, health officials in Canada would have had the option of initiating communication with health officials in the country where the threat was reported. Such lines of communication might involve requests for additional information to better characterize the threat, offers of international assistance and other operational matters. If needed, public health authorities, health care providers and members of the public at the site of the gathering could be alerted to facilitate early detection of new cases.

\section{Next steps}

The capability to integrate knowledge of worldwide patterns of commercial air travel and infectious disease surveillance in real time could significantly enhance global situational awareness of infectious disease threats. This information may have added value, in that it would complement and not compete with existing preparedness efforts at mass gatherings, it may be transferable to settings other than mass gatherings, it would harmonize with the core principles of the revised International Health Regulations, ${ }^{14}$ and, in the spirit of the Olympic Games, it could help to foster a culture of greater international cooperation in confronting global infectious disease threats of tomorrow. ${ }^{15}$

However, to explore this potential, additional research is needed to better understand mass gatherings and their interaction with global infectious diseases. Although historical events support the notion that mass gatherings may act as local amplifiers and global disseminators of infectious diseases, further validation of the principles described in our model is needed. The forthcoming G-20 summit in Ontario and the concurrent FIFA World Cup competition in South Africa in June 2010 may offer such opportunities. Equally important, further dialogue is needed between scientists at academic institutions and public health decision-makers in government to determine how best to translate knowledge of this kind into actionable intelligence that can be readily incorporated into decision-making routines.

\section{This article was peer reviewed.}

Competing interests: Kamran Khan founded Bio.DIASPORA and developed it with assistance from his staff Jun Wang, Wei Hu, David Kossowsky, Jennifer Sears and Angie Chan. The Bio.DIASPORA project has been supported by three public agencies: the Public Health Agency of Canada, the Ontario Ministry of Health and Long-Term Care, and St. Michael's Hospital, Toronto, Ont. John Brownstein and Clark C. Freifeld cofounded HealthMap and developed it with assistance from Sumiko R. Mekaru and Amy L. Sonricker. HealthMap has received unrestricted research grants from Google.org and the US National Library of Medicine, part of the US National Institutes of Health.

Contributors: Kamran Khan developed the conceptual model, performed statistical and network analyses, and wrote and edited the manuscript. Clark C. Freifeld developed the system for automated data acquisition from RSS feeds, performed initial keyword-based filtering of articles, and was involved in reviewing and editing the manuscript. Jun Wang performed statistical analyses of the data, and reviewed and edited the manuscript. Sumiko R. Mekaru participated in conceptual development of the use of RSS feeds, developed a catalogue for RSS feeds and selection of key words, reviewed disease alerts, and wrote and edited the manuscript. David Kossowsky developed spatial visualizations of the data, and reviewed and edited the manuscript. Amy L. Sonricker performed the retrospective analysis of disease 
alerts from English, Chinese and Spanish news feeds, assisted with compilation of RSS feeds from Spanish and Chinese sources, and was involved in reviewing and editing the manuscript. Wei Hu performed statistical and network analyses of the data and was involved in reviewing and editing the manuscript. Jennifer Sears participated in development of the conceptual model, reviewed the medical and public health literature, and was involved in reviewing and editing the manuscript. Angie Chan participated in development of the conceptual model, reviewed key government documents and considered their health policy implications, and was involved in reviewing and editing the manuscript. John S. Brownstein developed the conceptual model, acquired and interpreted the surveillance data, and was involved in writing and editing the manuscript. All authors approved the final version submitted for publication.

Funding: This work was supported by grant G08LM009776-01A2 from the US National Library of Medicine, the US National Institutes of Health, a research grant from Google.org and the Public Health Agency of Canada.

\section{REFERENCES}

1. Barbeschi M, Healing T. Communicable disease alert and response for mass gatherings: key considerations. Geneva (Switzerland): World Health Organization 2008. Available: www.who.int/csr/Mass gatherings2.pdf (accessed 2010 Feb. 22).

2. International consultation: infectious disease prevention and control at Umra and Hajj (2009) [technical meeting report]. Kingdom of Saudi Arabia, Ministry of Health; World Health Organization, Regional Office for the Eastern Mediterranean; Centers for Disease Control and Prevention; 2009. Available: www.emro .who.int/csr/h1n1/pdf/infectiousdiseases_hajj_umra.pdf (accessed 2010 Feb. 22).

3. Memish ZA, McNabb SJ, Mahoney F, et al. Establishment of public health security in Saudi Arabia for the 2009 Hajj in response to pandemic influenza A H1N1. Lancet 2009;374:1786-91.

4. Khan K, Memish ZA, Chabbra A, et al. Global public health implications of a mass gathering in Mecca, Saudi Arabia during the midst of an influenza pandemic. J Travel Med 2010. DOI 10.1111/j.1708-8305.2009.00391.x

5. Wilder-Smith A, Goh KT, Barkham T, et al. Hajj-associated outbreak strain of
Neisseria meningitidis serogroup W135: estimates of the attack rate in a defined population and the risk of invasive disease developing in carriers. Clin Infect Dis 2003;36:679-83

6. Khan K, Arino J, Calderon F, et al. The BIO.DIASPORA Project: an analysis of Canada's vulnerability to emerging infectious disease threats via the global airline transportation network. Toronto (ON): St. Michael's Hospital; 2009. Available: www.biodiaspora.com (accessed 2010 Feb. 22).

7. Khan K, Arino J, Hu W, et al. Spread of a novel influenza A (H1N1) virus via global airline transportation. N Engl J Med 2009;361:212-4.

8. Brownstein JS, Wolfe CJ, Mandl KD. Empirical evidence for the effect of airline travel on inter-regional influenza spread in the United States. PLoS Med 2006;3:e401.

9. Brownstein JS, Freifeld CC, Madoff LC. Digital disease detection - harnessing the Web for public health surveillance. N Engl J Med 2009;360:2153-7.

10. Wilson K, Brownstein JS. Early detection of disease outbreaks using the Internet. CMAJ 2009;180:829-31.

11. Bitar D, Goubar A, Desenclos JC. International travels and fever screening during epidemics: a literature review on the effectiveness and potential use of non-contact infrared thermometers. Euro Surveill 2009;14(6):pii:19115.

12. Brownstein JS, Freifeld CC, Reis BY, et al. Surveillance Sans Frontières: Internetbased emerging infectious disease intelligence and the HealthMap project. PLoS Med 2008;5:e151.

13. Ehresmann KR, Hedberg CW, Grimm MB, et al. An outbreak of measles at an international sporting event with airborne transmission in a domed stadium. $J$ Infect Dis 1995;171:679-83.

14. International health regulations. 2nd ed. Geneva (Switzerland); World Health Organization; 2005 [reprinted 2008]. Available: www.who.int/csr/ihr/en/ (accessed 2010 Feb. 22).

15. Prentice T, Reinders LT. The world health report 2007 - A safer future: global public health security in the 21st century. Geneva (Switzerland): World Health Organization; 2007. Available: www.who.int/whr/2007/en/index.html (accessed 2010 Feb. 22).

Correspondence to: Dr. John S. Brownstein, Children's Hospital Boston, Harvard Medical School, 1 Autumn St., Rm. 451, Boston

MA 02215; john_brownstein@harvard.edu

\section{***THIS NOTICE TO HEALTH CARE PROFESSIONALS IS SENT BY ORDER OF THE SUPERIOR COURT OF QUEBEC***}

\section{SUBJECT: AUTHORIZATION OF A CLASS ACTION WITH REGARD TO THE USE OF DEPO-PROVERA}

Notice to health care professionals.

Dear Health Care Professional,

On May 28, 2008, the Superior Court of Quebec authorized the institution of a class action against Pfizer Canada Inc. and Pfizer Inc., in Court file \# 500-06-000305-058, Brito v. Pfizer Canada Inc. and Pfizer Inc., on behalf of all persons that are included in the class defined as follows:

"Every person domiciled in Canada who claims to be suffering or to have suffered a loss of bone mineral density owing to the use of DEPO-PROVERA." (the "Class")

It is important that Class members be informed of the existence of this class action and of the possibility to opt-out of the proceeding, notably in order to institute separate proceedings. Your collaboration with regards to the dissemination of this information will contribute to notify the greatest number of Class members of the possibility for them to benefit from or to opt-out of the class action.

The opt-out date is May 31, 2010. After this date, every Class member who did not opt-out will be bound by any judgment or settlement in the class action. A Class member should not opt out if he or she wishes to participate in the class action. A Class member who opts out will not be eligible for any recovery in the class action.

For these reasons, you are kindly asked to notify without delay any of your patients who use or have used DEPO-PROVERA of the existence of this class action and to invite them to visit the website www.depoprovera.ca for more information on this class action and notably on the opt-out procedure.

If you have any questions, do not hesitate to contact the Representative plaintiff's counsel by email, fax or telephone at:

Belleau Lapointe, L.L.P.

Telephone: 1-888-987-6701 • Fax: (514) 987-6886

Email: info@belleaulapointe.com•www.depoprovera.ca 